\title{
The post-transplant scoring system (PTSS) is associated with outcomes in patients with MDS after CD34+selected allogeneic stem cell transplant
}

Ana Alarcon Tomas (iD) ${ }^{1}$, Karissa Whiting ${ }^{2}$, Molly Maloy ${ }^{1}$, Josel D. Ruiz ${ }^{1}$, Sean Devlin², Miriam Sanchez-Escamilla ${ }^{3,4}$, Lucrecia Yañez ${ }^{3,4}$, Nerea Castillo ${ }^{1}$, Martina Pennisi ${ }^{1,5}$, Christina Cho ${ }^{1,6}$, Brian Shaffer ${ }^{1,6}$, Hugo Castro-Malaspina ${ }^{1,6}$, Virginia Klimek ${ }^{6,7}$, Sergio A. Giralt (iD ${ }^{1,6}$, Roni Tamari ${ }^{1,6}$ and Miguel-Angel Perales (iD) ${ }^{1,6 凶}$

(c) The Author(s), under exclusive licence to Springer Nature Limited 2021

The post-transplant scoring system (PTSS), developed by the Francophone Society of Bone Marrow Transplantation and Cellular Therapy, is based on three independent post-transplant risk factors: grade of acute graft-versus-host disease, lack of platelet recovery before day 100, and relapse before day 100; discriminating low- (0), intermediate- (1-3), and high-risk (4-8) patients. We investigated the prognostic value of the PTSS in a cohort of patients with MDS who underwent myeloablative CD34-selected TCD transplants. From 2008 to 2018, 109 patients underwent a first TCD-HCT for MDS at our center. We used Cox proportional hazards models and different landmark analyses to evaluate the association of categorized PTSS score risk groups with overall survival (OS). Patients with an intermediate/ high risk PTSS score had decreased OS at day 180 (univariate HR 3.25 [95\% Cl 1.60, 6.60], $p=0.001$ ) and at day 365 (univariate $\mathrm{HR} 5.42$ [95\% Cl 2.21, 13.3], $p<0.001$ ) compared to low risk PTSS scores. This association remained significant after adjusting for HCT-Cl. PTSS score calculated at day 100 was not associated with OS, even after adjusting for HCT-CI subgroups. In summary, the PTSS predicted survival at day 180 and day 365 in recipients of T-cell-depleted allografts for myelodysplastic syndrome.

Bone Marrow Transplantation (2021) 56:2749-2754; https://doi.org/10.1038/s41409-021-01392-w

\section{INTRODUCTION}

Myelodysplastic syndrome (MDS) comprises a very heterogeneous group of myeloid neoplasms with varying outcomes. While several therapies have been developed in recent years, allogeneic hematopoietic stem cell transplantation $(\mathrm{HCT})$ remains the only potentially curative treatment for these patients. However, its use is restricted due to the risk of morbidity and mortality [1]. In the past decade, as a result of advances in therapy and supportive care, along with wider donor availability, the number of patients with MDS undergoing HCT has been increasing [2].

Graft-versus-host disease (GVHD) remains one of the main causes of transplant-related morbidity and mortality. One strategy to reduce GVHD is the use of ex vivo T-cell depletion (TCD) of the graft through CD34+ selection. We have previously reported rates of overall survival (OS) of $56.9 \%$ at 2 years $(95 \%$ $\mathrm{Cl}, 48-67.3 \%)$ and $49.3 \%$ at 5 years $(95 \% \mathrm{Cl}, 40.4-60.2 \%)$ with cumulative incidences of grades II-IV acute GVHD of $9.8 \%$ at day 100 in patients with MDS undergoing this approach [3]. This study demonstrated that CD34-selected HCT offers longterm OS and RFS with low rates of acute and chronic GVHD, and without an increased risk of relapse.
Since outcomes for patients with MDS are heterogeneous, individual risk stratification is important in managing patients. Most published scoring systems have focused on prognostic variables measured before HCT. The International Prognostic Scoring System (IPSS) [4] and the revised IPSS (IPSS-R) [5] are commonly used by clinicians to help risk stratification. While these scores are helpful to guide decisions prior to transplant, they are less useful in predicting outcomes in patients who have already undergone HCT. Post-transplant complications are well known and can be easily identified, but no definitive prediction model based on them exits. Robust statistical models of post-transplant complications should guide decisions for each patient with a riskadapted individualized strategy.

The Francophone Society of Bone Marrow Transplantation and Cellular therapy (SFGM-TC) group has recently validated a scoring system based on post-transplant complications that can estimate the survival probability of patients with MDS who survive more than 100 days after HCT [6]. This post-transplant scoring system (PTSS) is a clinical risk assessment tool based on three independent risk factors: grade of acute GVHD, lack of platelet recovery before day 100 , and relapse before day 100 . This score

\footnotetext{
${ }^{1}$ Adult Bone Marrow Transplant Service, Department of Medicine, Memorial Sloan Kettering Cancer Center, New York, NY, USA. ${ }^{2}$ Department of Biostatistics and Epidemiology, Memorial Sloan Kettering Cancer Center, New York, NY, USA. ${ }^{3}$ Department of Hematology, University Hospital Marqués de Valdecilla, Santander, Spain. ${ }^{4}$ Department of Hematological Malignancies and Stem Cell Transplantation, Research Institute of Marques de Valdecilla (IDIVAL), Santander, Spain. ${ }^{5}$ Department of Oncology and HematoOncology, University of Milan, Milan, Italy. ${ }^{6}$ Department of Medicine, Weill Cornell Medical College, New York, NY, USA. ' ${ }^{7}$ eukemia Service, Department of Medicine, Memorial Sloan Kettering Cancer Center, New York, NY, USA. ${ }^{凶}$ email: peralesm@mskcc.org
} 
can be helpful in decision making for patients with complications after allo-HCT.

In this retrospective study, we calculated the PTSS at different timepoints after transplant in a cohort of patients with MDS who underwent TCD-HCT, in an attempt to validate the prognostic tool using a different transplant approach.

\section{MATERIAL AND METHODS \\ Patients}

The analysis included patients aged 18 or older diagnosed with MDS who underwent a first allo-HCT using ex vivo CD34+ cell-selected peripheral blood stem cell (PBSC) transplant. Patients were treated at Memorial Sloan Kettering Cancer Center between January 1, 2008 and May 31, 2018. Patients with MDS classified by WHO category 2008 [7] were included. Disease status at transplant (responder vs no responder) was assessed according to IWG 2006 criteria. HLA matching was established by DNA sequence-specific oligonucleotide typing for HLA-A, - B, -C, -DRB1, and -DQB1 loci. Patients received grafts from HLA-matched (10/10) or single mismatched (9/10) related or unrelated donors. Clinical outcomes, including acute GVHD, engraftment, relapse, and causes of death, were captured per standard clinical practice. Written informed consent for treatment was obtained from all patients and donors. Approval for this retrospective review was obtained from the Institutional Review Board and Privacy Board.

\section{Conditioning regimens and graft source}

All patients were treated with myeloablative conditioning regimens, including hyper fractionated total body irradiation 1375 CGy over 4 days, followed by thiotepa $5 \mathrm{mg} / \mathrm{kg} /$ day i.v. for 2 days and either fludarabine 25 $\mathrm{mg} / \mathrm{m}^{2} /$ day i.v. for 5 days, or cyclophosphamide $60 \mathrm{mg} / \mathrm{kg} /$ day i.v. for 2 days; or busulfan followed by melphalan $70 \mathrm{mg} / \mathrm{m}^{2} /$ day i.v. for 2 days, and fludarabine $25 \mathrm{mg} / \mathrm{m}^{2} /$ day i.v. for 5 days. TCD of granulocyte colonystimulating factor-mobilized PBSCs grafts was performed as described previously $[8,9]$. Ex vivo CD34+ selection of hematopoietic progenitor cells was performed using one of two methods as previously described: Isolex 300i Magnetic Cell Separator (Baxter, Deerfield, IL), followed by T cell rosetting with sheep erythrocytes (9 patients), or using the CliniMACS ${ }^{\circ}$ CD34+ Reagent System (Miltenyi Biotech, Gladbach, Germany). All patients received either equine or rabbit anti-thymocyte globulin (ATG). Patients did not receive any other post-transplant immunosuppressive prophylaxis. All patients received supportive care and prophylaxis against opportunistic infections according to standard guidelines [10].

\section{Post-transplant scoring system (PTSS) and hematopoietic cell transplantation comorbidity index (HCT-CI)}

The PTSS score assigns 1 point for grade II acute GVHD, 2 points for grade III/IV acute GVHD, 2 points for lack of platelets recovery before day 100, and 4 points for relapse. GVHD was diagnosed clinically, confirmed pathologically whenever possible, and classified according to standard criteria $[11,12]$. Lack of platelets recovery until day 100 was defined as the absence of platelets $>20,000 / \mu l$ or need for transfusion until day 100 . Relapse or progression was identified by conventional diagnostic methods. Classification of patients into risk groups was the same as the original study [6]: the point score model ranged from 0 to 8 points, discriminating low- (0), intermediate- (1-3), and high-risk (4-8) patients. PTSS was also analyzed as a two-level variable with patients with scores $<1$ categorized as low, and patients $\geq 1$ categorized as intermediate/high. The HCT-Cl was calculated as originally described $[13,14]$ and patients were classified in two ways: (1) low (0-1), intermediate (2-3), and high (>3) and (2) low/ intermediate $(0-3)$ and high $(>3)$.

\section{Endpoints and statistical analysis}

The primary endpoint of the study was OS, defined as time from landmark date $(100,180$, or 365 days post HCT) until death from any cause or the last follow-up. Kaplan-Meier curves were used to estimate event-time distribution for OS endpoint. To evaluate the association between PTSS score and OS, a landmark analysis was performed at day 100, 180, and 365 post HCT. Patients who died before the landmark timepoint (death before day 100,180 , and 365 ) were excluded. To evaluate the association between the $\mathrm{HCT}-\mathrm{Cl}$ and $\mathrm{OS}$, univariate cox proportional hazards models were fit from landmark timepoints at baseline (day 0) and day 100, 180, and 365. For exploratory purposes, multivariate cox proportional hazard models were used to investigate if PTSS score was prognostic after adjusting for the $\mathrm{HCT}-\mathrm{Cl}$. Univariate and multivariate cox proportional hazard models were fit using each respective landmark timepoint as baseline to obtain hazard ratio (HR) estimates and corresponding confidence intervals (Cls) of OS. Secondary endpoints included cumulative incidence of relapse and cumulative incidence of acute GvHD. Cumulative incidence of relapse was estimated considering non-relapse mortality (NRM) as a competing risk. Cumulative incidence of acute GVHD ( $\geq$ grade 2 ) was estimated with death without prior relapse, or relapse considered as competing risk events. Descriptive statistics were used to summarize patient characteristics. All statistical analyses were performed using $\mathrm{R}$ version 3.6.2.

\section{RESULTS}

\section{Patients' characteristics}

One hundred and nine patients with MDS met the inclusion criteria and constituted the study population. Median age was 61 years (range 20-72), 64 patients (59\%) were male, and $89(82 \%)$ had a matched related or unrelated donor. Table 1 describes the baseline characteristics and early post-transplant complications of the cohort.

Patients were distributed per the WHO classification 2008: refractory anemia with excess of blasts type 2 (RAEB-2) $=28$ $(25.7 \%)$, refractory anemia with excess of blasts type 1 (RAEB-1) $=$ $34(31.2 \%)$, refractory cytopenia with unilineage dysplasia (RCUD), refractory anemia with ringed sideroblasts (RARS), refractory cytopenia with multilineage dysplasia $(\mathrm{RCMD})=39(35.8 \%), \mathrm{MDS}$ with isolated del(5q) 4 (3.7\%), and unclassified $=4(3.7 \%)$.

Most patients had received prior hypomethylating agents (decitabine or azacitidine), including six patients treated on a clinical trial of ATRA + decitabine (NCT00382200). Patients with del5q and transfusion-dependent anemia were also treated with lenalidomide, and one patient received ATG. The remaining patients proceeded directly to transplant, as they were classified as high risk by cytogenetics with $<10 \%$ blasts in bone marrow. Over $70 \%$ patients responded to the pre-HCT treatments and most had less than $5 \%$ bone marrow blasts pre-HCT, although only 12 patients achieved a complete remission pre-transplant.

\section{Engraftment and graft-versus-host disease}

Successful engraftment was observed in 107 patients. Only 2 patients (1.8\%) did not recover their platelet count before day 100. The cumulative incidence of grade II-IV acute or late acute GVHD at day 100 and day 180 were $19 \%(95 \% \mathrm{Cl} 12-27 \%)$ and $24.84 \%$ (95\% Cl 17-33\%), respectively (Fig. 1a). Before the 100-day landmark, 22 patients had developed grade I, 13 had developed grade II, and 3 grade III GVHD. Before the 1-year landmark, 20 patients had developed grade I, 13 grade II, and 11 grade III-IV GVHD. The remaining patients $(n=65)$ had no GVHD at 1 year.

\section{Relapse/progression and non-relapse mortality}

The 3-year cumulative incidence of relapse was $17.1 \%(95 \% \mathrm{Cl}$ $10.5-24.9 \%$ ) with a median time to relapse after transplant of 9 months (range, 3-90 months). Among 23 patients with relapse events, 6 were alive at last follow-up (Fig. 1b). Among patients who relapsed during the first year, one relapsed before day 100, 4 before day 180 landmark, and 14 before day 365 . The 3-year cumulative incidence of NRM was $20 \%$ (95\% Cl 13-28\%). At last follow-up, relapse was the most common cause of death, GVHD was the second most common cause of death, followed closely by infection.

\section{Pre-transplant HCT-Cl score and PTSS}

The median HCT-Cl score was 2. Thirty-six patients (33\%) had a low, 40 (37\%) an intermediate, and 33 (30\%) a high HCT-Cl score. Twenty-one patients had an HCT-Cl score $\geq 5$ (19.3\%). Sixteen of the 17 comorbidities included in the $\mathrm{HCT}-\mathrm{Cl}$ were present in at 
Table 1. Characteristics of the transplant and post-transplant scoring system complications.

\begin{tabular}{|c|c|c|}
\hline & & $\begin{array}{l}\text { Cohort } \\
(n=109)\end{array}$ \\
\hline \multicolumn{3}{|l|}{ Characteristics of the transplant } \\
\hline \multicolumn{2}{|l|}{ Recipient age, years, mean \pm SD } & $61 \pm 10.9$ \\
\hline \multirow[t]{2}{*}{ Recipient sex, $n(\%)$} & Male & $64(58.7)$ \\
\hline & Female & $45(41.3)$ \\
\hline \multicolumn{2}{|l|}{ Sex mismatched } & $45(41.3)$ \\
\hline \multirow[t]{5}{*}{ WHO category, $n$ (\%) } & RCUD/RARS/RCMD & $39(35.8)$ \\
\hline & RAEB-1 & $34(31.2)$ \\
\hline & RAEB-2 & $28(25.7)$ \\
\hline & Del5q & $4(3.7)$ \\
\hline & Unclassified & $4(3.7)$ \\
\hline \multirow[t]{2}{*}{ IPSS score at transplant, $n$ (\%) } & Low/intermediate 1 & $82(79.6)$ \\
\hline & Intermediate-high 2 & $21(20.4)$ \\
\hline \multirow[t]{3}{*}{$\begin{array}{l}\text { R-IPSS. Cytogenetic risk score at } \\
\text { transplant, } n(\%)\end{array}$} & $\begin{array}{l}\text { Favorable } \\
\text { (low, very low) }\end{array}$ & $60(58.3)$ \\
\hline & Intermediate & $23(22.3)$ \\
\hline & $\begin{array}{l}\text { High risk (high, } \\
\text { very high) }\end{array}$ & $20(19.4)$ \\
\hline \multirow[t]{4}{*}{ Pre-transplantation therapy, $n$ (\%) } & $\begin{array}{l}\text { Hypomethylating } \\
\text { agents only }\end{array}$ & $84(77.1)$ \\
\hline & Chemotherapy only & $0(0)$ \\
\hline & Both & $6(5.5)$ \\
\hline & None & $19(18.4)$ \\
\hline \multirow[t]{2}{*}{ Disease status at transplant, $n(\%)$} & Responder & $64(58.7)$ \\
\hline & No responder & $45(41.3)$ \\
\hline \multirow[t]{3}{*}{ Bone marrow blast count, $N(\%)$} & $<5 \%$ & $85(82.5)$ \\
\hline & $\geq 5 \%$ & $15(14.6)$ \\
\hline & Not evaluable & $3(2.9)$ \\
\hline \multirow[t]{2}{*}{ HLA matching, $n(\%)$} & No & $20(18.3)$ \\
\hline & Yes, MRD & $\begin{array}{l}89(81.7), \\
36(33)\end{array}$ \\
\hline \multirow[t]{3}{*}{ Donor CMV status, $n$ (\%) } & Positive & $40(36.7)$ \\
\hline & Negative & $68(62.4)$ \\
\hline & Inconclusive & $1(0.92)$ \\
\hline \multirow[t]{2}{*}{ Recipient CMV status, $n$ (\%) } & Positive & $50(45.9)$ \\
\hline & Negative & $59(54.1)$ \\
\hline \multirow[t]{2}{*}{ Total body irradiation, $n(\%)$} & No & $103(94.5)$ \\
\hline & Yes & $6(5.5)$ \\
\hline \multicolumn{3}{|c|}{ Post-transplant scoring system complications } \\
\hline \multirow{3}{*}{$\begin{array}{l}\text { Grade of acute GVHD until day } \\
100, n(\%)\end{array}$} & $0 / l$ & $87(84.5)$ \\
\hline & II & $13(12.6)$ \\
\hline & III/IV & $3(2.9)$ \\
\hline \multirow{3}{*}{$\begin{array}{l}\text { Grade of acute GVHD until day } \\
180, n(\%)\end{array}$} & $0 / 1$ & $85(78)$ \\
\hline & II & $13(11.9)$ \\
\hline & III/IV & $11(10.1)$ \\
\hline \multirow{3}{*}{$\begin{array}{l}\text { Grade of acute GVHD until day } \\
180, n(\%)\end{array}$} & $0 / 1$ & $85(78)$ \\
\hline & II & $13(11.9)$ \\
\hline & III/IV & $11(10.1)$ \\
\hline \multirow{2}{*}{$\begin{array}{l}\text { Lack of platelet recovery day } 100, \\
n(\%)\end{array}$} & No & $107(98.1)$ \\
\hline & Yes & $2(1.8)$ \\
\hline
\end{tabular}

Table 1 continued

\begin{tabular}{lll}
\hline & & $\begin{array}{l}\text { Cohort } \\
(\boldsymbol{n}=109)\end{array}$ \\
\hline Relapse before day $100, n(\%)$ & No & $108(99.1)$ \\
\hline Relapse before day $180, n(\%)$ & Yes & $1(0.9)$ \\
\hline Relapse before day $365, n(\%)$ & Yes & $104(96.2)$ \\
\hline & Yes & $4(3.7)$ \\
\hline
\end{tabular}

CMV cytomegalovirus, WHO World Health Organization.

least one patient, except for renal comorbidity. The most frequent comorbidities included pulmonary impairment (moderate, $n=21$; severe, $n=15)$, prior solid tumor (22 patients, 19.3\%), psychiatric disorders (21 patients, 19.3\%), and mild hepatic impairment (19 patients, $17.5 \%)$. Since the HCT-Cl was only assessed at baseline, we also examined the KPS at the landmark timepoints (day 180 and day 365). At day 180, KPS ranged from 20 to 90, with 30 of 102 patients (30\%) having a KPS $<70$ and 71 patients (70\%) having KPS $>70$. KPS was missing in one patient. At day 365 , KPS ranged from 30 to 100, with 17 of 90 patients (19\%) having a KPS $<70$ and 73 patients $(81 \%)$ having KPS $>70$.

At day 100,90 patients $(82.6 \%)$ had a low risk PTSS score, 18 patients $(16.5 \%)$ an intermediate risk, and only 1 patient $(0.9 \%)$ was classified as high risk. At post-transplant day 180, 72 of 102 patients at this time $(71.3 \%)$ had a PTSS of low risk, 25 patients (24.5\%) an intermediate score, and 4 patients (3.9\%) a high-risk score. The score was missing in one patient at this timepoint. At the day 365 landmark, 55 of 90 patients still on study were low risk (61.1\%), 26 patients (28.9\%) were intermediate risk, and $9(10.0 \%)$ were high risk.

\section{Overall survival}

With a median follow-up of 59 months $(95 \% \mathrm{Cl} 56,66), 1-$ and 3year OS in the overall population were $85 \%(95 \% \mathrm{Cl} 79,92)$ and $67 \%(95 \% \mathrm{Cl} 58,77)$, respectively. Seventy patients $(64.2 \%)$ were alive at last follow-up. No patients died or were lost to follow-up before day 100 . Six patients died, one patient was lost to follow-up before the 180-day landmark timepoint, and one patient did not have data available for scoring at day 180. By 365 days, ten additional patients died and two were lost to follow-up. Among the 55 low-risk patients who were included in the 365-day landmark analysis, the median follow-up was 65.6 months $(95 \% \mathrm{Cl}$ 58.8-76.2 months) from time of transplant. Eight patients in this group died after day 365 . The range of follow-up among those who did not have an event is 16.4-114.7 months from transplant.

OS did not significantly differ between HCT-Cl groups at baseline (day 0; log rank $p=0.53$; Fig. 2). Similarly, there was no association between HCT-Cl and OS at any of the other landmark timepoints (day 100, 180, and 365) (Table 2). Additional analysis using a modified two-group scoring system of low-risk (0-3) and high-risk (>3) HCT-Cl groups did not show any difference.

Due to limited events, we categorized KPS into two groups $(\leq 70$ and $>70$ ) to limit multivariate models to three variables. There was no association between KPS and OS and day 180 (HR $0.63[95 \% \mathrm{Cl}$ $0.30-1.32] p$ value $=0.2$ ), but there was a significant association at day 365 (HR 0.24 [95\% Cl 0.10-1.57] $p$ value $=0.001)$. Overall, the strength and direction of associations do not change greatly after adjusting for KPS at these timepoints.

Because of limited number of events in some of the landmark groups, PTSS was analyzed as a two-level variable: low (0), and intermediate-high $(\geq 1)$. Using the PTSS at day 180 , OS was significantly lower in the intermediate/high-risk group (HR 3.25 $[95 \% \mathrm{Cl} 1.60,6.60] p$ value 0.001 ) (Fig. 3a) compared to the low-risk 

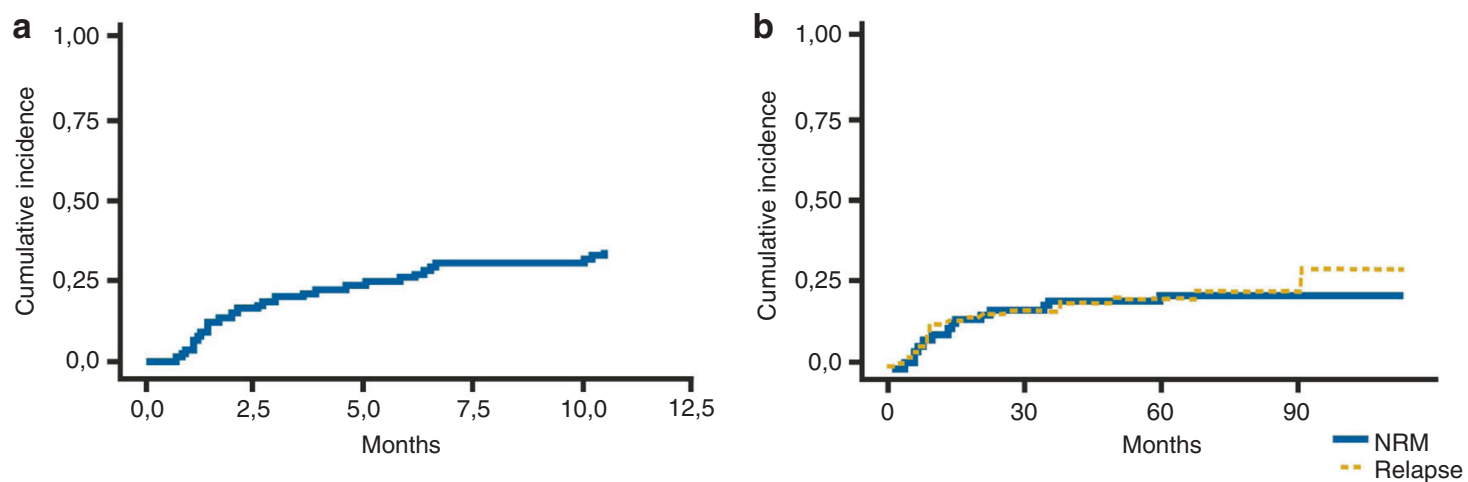

Fig. 1 Transplant outcomes in patients with MDS. a Cumulative incidence of GVHD grade II-IV. b Cumulative incidences of relapse and NRM.

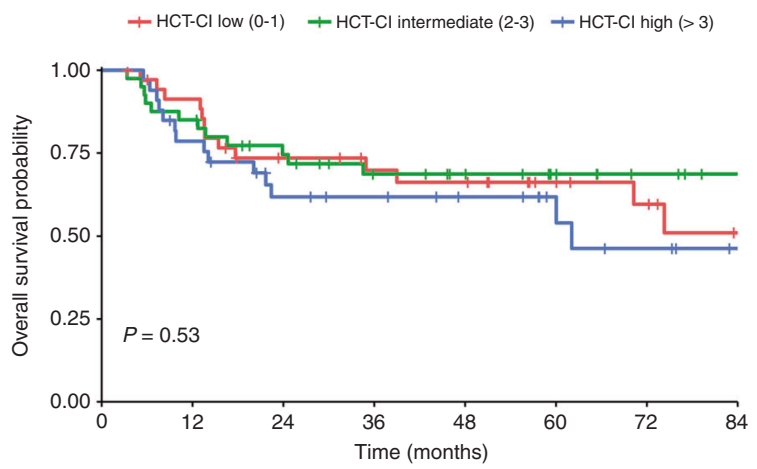

Fig. 2 Overall survival in patients with MDS based on $\mathrm{HCT}-\mathrm{Cl}$ subgroups at baseline.

Table 2. Univariate cox proportional hazard models.

\begin{tabular}{|c|c|c|c|c|}
\hline \multirow[t]{2}{*}{ Landmark } & \multirow[t]{2}{*}{ Score subgroups } & \multicolumn{3}{|c|}{ Overall survival } \\
\hline & & HR & $95 \% \mathrm{Cl}$ & $p$ value \\
\hline \multirow[t]{4}{*}{ Day 0-Baseline } & $\mathrm{HCT}-\mathrm{Cl}$ & & & \\
\hline & Low (0-1) & - & - & \\
\hline & Intermediate (2-3) & 0.83 & $0.38,1.81$ & 0.6 \\
\hline & High $(>3)$ & 1.28 & $0.60,2.73$ & 0.5 \\
\hline \multirow[t]{4}{*}{ Day 100} & $\mathrm{HCT}-\mathrm{Cl}$ & & & \\
\hline & Low $(0-1)$ & - & - & \\
\hline & Intermediate (2-3) & 0.83 & $0.38,1.81$ & 0.6 \\
\hline & High $(>3)$ & 1.28 & $0.60,2.73$ & 0.5 \\
\hline \multirow[t]{4}{*}{ Day 180} & $\mathrm{HCT}-\mathrm{Cl}$ & & & \\
\hline & Low $(0-1)$ & - & - & \\
\hline & Intermediate (2-3) & 0.6 & $0.24,1.46$ & 0.3 \\
\hline & High $(>3)$ & 1.32 & $0.60,2.89$ & 0.5 \\
\hline \multirow[t]{4}{*}{ Day 365} & $\mathrm{HCT}-\mathrm{Cl}$ & & & \\
\hline & Low $(0-1)$ & - & - & \\
\hline & Intermediate (2-3) & 0.53 & $0.19,1.46$ & 0.2 \\
\hline & High (>3) & 0.89 & $0.34,2.33$ & 0.8 \\
\hline
\end{tabular}

$H R$ hazard ratio, $\mathrm{Cl}$ confidence interval.

Univariable analysis that shows the association of HCT-Cl subgroups and OS at different landmark timepoints (day 100, 180, and 365).

group. Similarly, when assessed at day 365 intermediate/high-risk group, PTSS was significantly associated with shorter OS (HR 5.42 $[95 \% \mathrm{Cl} 2.21,13.3] p$ value $<0.001)$ compared to the low-risk group (Fig. 3b). PTSS score calculated at day 100 was not associated with survival (HR of intermediate/high-risk group: 1.89 [0.89, 4.01] $p$ value 0.10$)$. In the multivariate analyses, intermediate/high PTSS scores remained significantly associated with shorter OS, after adjusting for comorbidity index (Table 3 ).

\section{DISCUSSION}

There are limited data regarding post-transplant prognostic evaluation [15-17]. The SFGM-TC group proposed and validated the PTSS, showing that post-transplant complications were the only independent risk factors associated with decreased OS, while this was not the case for pre-transplant factors. In our cohort of 109 patients with MDS who received myeloablative CD34-selected $\mathrm{HCT}$, patients with higher PTSS scores at 6 months and 1 year after transplant had a significantly lower OS compared to patients with lower scores, thus confirming the results of the original study. Patients included in the study had similar baseline characteristics to those in the French study in terms of age, gender, WHO category, pre-transplantation therapy, disease status at transplant, and donor and recipient CMV status. In addition to using a different transplant approach, other differences included a higher number of patients with less than $5 \%$ blasts in the bone marrow at time of transplant (82\%) and higher number of HLA matching in our population.

The PTSS was originally developed to estimate the survival probability of patients with MDS who survive more than 100 days after HCT. However, the day 100 landmark PTSS was not associated with OS in our patient cohort. Possible explanations for these differences include the fact that we observed a lower incidence of acute and chronic GVHD in the TCD group compared to what is seen in unmodified grafts [18-24]. We have previously reported cumulative incidences of grades II-IV acute GVHD of $9.8 \%$ at day 100 and $15.7 \%$ at day 180 in patients with advanced MDS who received a CD34-selected allo-HCT [3]. In contrast in the French study, $38.2 \%$ of the patients had a grade II-IV GVHD before day 100 , while only $19 \%$ had grade II-IV GVHD at day 100 in our cohort.

Furthermore, the use of myeloablative conditioning regimens in recipients of CD34-selected transplants results in a significantly lower incidence of overall relapse and progression. In the French study, $8.9 \%$ patients relapsed before day 100 while just one patient $(0.9 \%)$ relapsed or progressed in our study at the same timepoint. These significant differences in both GVHD and relapse may account for the fact that the day 100 score was not correlated with survival in the TCD group.

Interestingly, the HCT-Cl was not predictive of OS in our study, even when we used a modified two-group scoring system of lowrisk (0-3) and high-risk (>3) HCT-Cl groups, which has been used in previous studies to stratify patients within a small sample size. The HCT-Cl score is routinely used in clinical practice and has been extensively validated as a robust predictor of NRM and survival, 
a

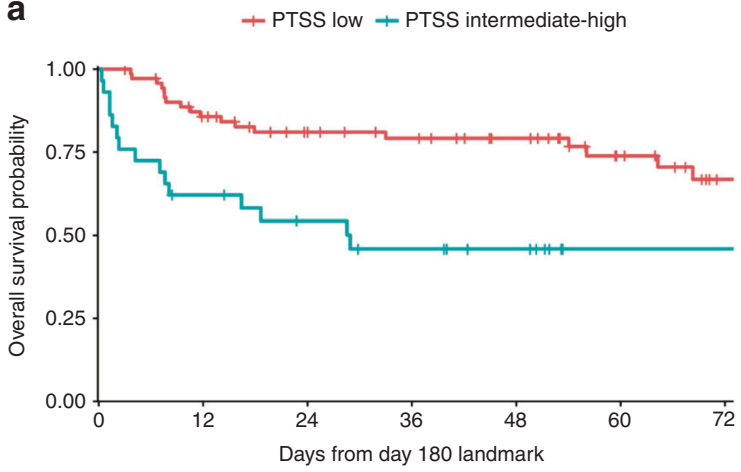

b

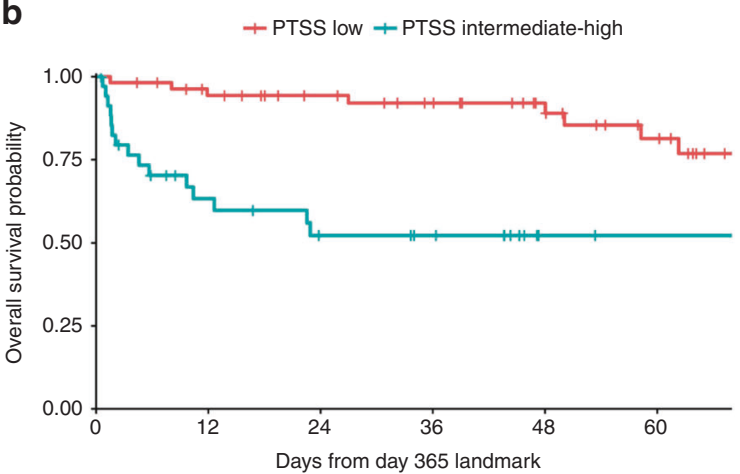

Fig. 3 Overall survival in patients with MDS based on PTSS subgroups. a OS with landmark analysis at day 180 . b OS with landmark analysis at day 365.

Table 3. Multivariate cox proportional hazard models.

\begin{tabular}{|c|c|c|c|c|}
\hline \multirow[t]{2}{*}{ Landmark } & \multirow[t]{2}{*}{ Score subgroups } & \multicolumn{3}{|c|}{ Overall survival } \\
\hline & & HR & $95 \% \mathrm{Cl}$ & $p$ value \\
\hline \multirow[t]{3}{*}{ Day 100} & PTSS & & & \\
\hline & Low & - & - & \\
\hline & Intermediate/high & 1.98 & $0.93,4.23$ & 0.077 \\
\hline \multirow[t]{3}{*}{ Day 100} & $\mathrm{HCT}-\mathrm{Cl}$ & & & \\
\hline & Low/intermediate & - & - & \\
\hline & High (>3) & 1.49 & $0.77,2.88$ & 0.2 \\
\hline \multirow[t]{3}{*}{ Day 180} & PTSS & & & \\
\hline & Low & - & - & \\
\hline & Intermediate/high & 3.55 & $1.73,7.28$ & $<0.001$ \\
\hline \multirow[t]{3}{*}{ Day 180} & $\mathrm{HCT}-\mathrm{Cl}$ & & & \\
\hline & Low/intermediate & - & - & \\
\hline & High $(>3)$ & 1.95 & $0.96,3.95$ & 0.066 \\
\hline \multirow[t]{3}{*}{ Day 365} & PTSS & & & \\
\hline & Low & - & - & \\
\hline & Intermediate/high & 5.89 & $2.35,14.7$ & $<0.001$ \\
\hline \multirow[t]{3}{*}{ Day 365} & $\mathrm{HCT}-\mathrm{Cl}$ & & & \\
\hline & Low/intermediate & - & - & \\
\hline & High $(>3)$ & 1.6 & $0.65,3.98$ & 0.3 \\
\hline
\end{tabular}

$H R$ hazard ratio, $\mathrm{Cl}$ confidence interval.

Multivariable analysis that shows the association of PTSS subgroups and OS at different landmark timepoints (day 100, 180, and 365) after adjusting for $\mathrm{HCT}-\mathrm{Cl}$ subgroups.

including in patients receiving CD34+-selected grafts $[25,26]$. Both of these prior reports from our center included larger cohorts of patients as well as patients with acute leukemia. While we cannot exclude that further discrimination would be detected in a larger cohort, a potential interpretation is that pre-existing comorbidities are less relevant in terms of OS in MDS patients who underwent TCD transplants.

Robust statistical models of post-transplant complications should guide decisions for each patient but for now, this new score can be helpful in making decisions after transplant. The prompt identification of high-risk PTSS patients should be targeted for interventions as soon as possible to reduce the likelihood of death. For example, strategies that mitigate relapse, such as the use of post-transplant hypomethylating agents, could be used in a risk-stratified manner based on the PTSS.

Similar to other landmark analyses, the PTSS can provide more accurate information on expected outcomes for patients who reach the landmark. Furthermore, the PTSS may also be helpful in making decisions in the setting of potentially life-threatening complications unrelated to transplant. For example, during the COVID-19 pandemic, patients, families, and the medical team are often faced with having to make decisions regarding withholding or withdrawing life support, or using aggressive and/or investigational treatments in patients who developed COVID-19 infection after a bone marrow transplant. The excellent survival in patients with a low PTSS score (estimated 3-year OS of 70\%) would argue for aggressive care in this setting.

The PTSS was originally developed in patients with MDS, and we now validate its use in a different transplant approach. However, it would be interesting to examine whether it may be applicable in other diseases such as AML. Although the multivariate model in the original study included several variables including early relapse, the only independent risk factors associated significantly with 3-year OS were the posttransplant complications. In contrast, early relapse in $A M L$ is typically associated with dismal survival. As a result, a score that includes relapse as well as post-HCT complications is likely to be of lesser prognostic value in AML. The more chronic nature of MDS may in part explain these likely differences. However, the value or lack of value of the PTSS in diseases other than MDS would need to be established in more formal studies.

Even though our study largely confirms the results of the previous study, there are certain limitations. The main one is the risk of selection or information bias that can be seen on in any retrospective study.

We conclude that, in patients with MDS undergoing TCD-HCT, the PTSS scores at day 180 and day 365 are significantly associated with OS. The lower incidence of acute GVHD in recipients of CD34selected transplants and the use of myeloablative condition regimens, with lower early relapse rates, likely explain differences with the original finding by the French group that the PTSS was predictive of survival at day 100 in unmodified grafts. Further studies including prospective evaluation are needed to validate these results, as well as the use of these tools in prospective clinical trials that seek to improve outcomes in patients with higher PTSS scores.

\section{REFERENCES}

1. Tamari R, Castro-Malaspina H. Transplant for MDS: challenges and emerging strategies. Best Pract Res Clin Haematol. 2015;28:43-54.

2. D'Souza A, Lee S, Zhu X, Pasquini M. Current use of and trends in hematopoietic cell transplantation in the United States. Biol Blood Marrow Transplant. 2017;23: 1417-21.

3. Tamari R, Chung SS, Papadopoulos EB, Jakubowski AA, Hilden P, Devlin SM, et al. CD34-selected hematopoietic stem cell transplants conditioned with myeloablative regimens and antithymocyte globulin for advanced myelodysplastic syndrome: limited graft-versus-host disease without increased relapse. Biol Blood Marrow Transplant. 2015;21:2106-14. 
4. Greenberg P, Cox C, LeBeau MM, Fenaux P, Morel P, Sanz G, et al. International scoring system for evaluating prognosis in myelodysplastic syndromes. Blood. 1997;89:2079-88.

5. Greenberg PL, Tuechler H, Schanz J, Sanz G, Garcia-Manero G, Solé F, et al. Revised International Prognostic Scoring System for myelodysplastic syndromes. Blood. 2012;120:2454-65.

6. Caulier A, Drumez E, Gauthier J, Robin M, Blaise D, Beguin Y, et al. Scoring system based on post-transplant complications in patients after allogeneic hematopoietic cell transplantation for myelodysplastic syndrome: a study from the SFGM-TC. Curr Res Transl Med. 2019;67:8-15.

7. Swerdlow SH, Campo E, Harris NL, Jaffe ES, Pileri SA, Stein $H$, et al. WHO classification of tumours of haematopoietic and lymphoid tissues 2008. Lyon, France: IARC.

8. Papadopoulos EB, Carabasi MH, Castro-Malaspina H, Childs BH, Mackinnon S, Boulad $\mathrm{F}$, et al. T-cell-depleted allogeneic bone marrow transplantation as postremission therapy for acute myelogenous leukemia: freedom from relapse in the absence of graft-versus-host disease. Blood. 1998;91:1083-90.

9. Jakubowski AA, Small TN, Young JW, Kernan NA, Castro-Malaspina H, Hsu KC, et al. T cell depleted stem-cell transplantation for adults with hematologic malignancies: sustained engraftment of HLA-matched related donor grafts without the use of antithymocyte globulin. Blood. 2007;110:4552-9.

10. Jakubowski AA, Small TN, Kernan NA, Castro-Malaspina H, Collins N, Koehne G, et al. $\mathrm{T}$ cell depleted unrelated donor stem cell transplantation provides favorable disease-free survival for adults with hematologic malignancies. Biol Blood Marrow Transplant. 2011;17:1335-42.

11. Rowlings PA, Przepiorka D, Klein JP, Gale RP, Passweg JR, Henslee-Downey PJ, et al. IBMTR Severity Index for grading acute graft-versus-host disease: retrospective comparison with Glucksberg grade. Br J Haematol. 1997;97:855-64.

12. Filipovich AH, Weisdorf D, Pavletic S, Socie G, Wingard JR, Lee SJ, et al. National Institutes of Health consensus development project on criteria for clinical trials in chronic graft-versus-host disease: I. Diagnosis and Staging Working Group Report. Biol Blood Marrow Transplant. 2005;11:945-56.

13. Sorror ML, How I. Assess comorbidities before hematopoietic. Cell Transplant. 2013;121:2854-63.

14. Sorror ML, Maris MB, Storb R, Baron F, Sandmaier BM, Maloney DG, et al. Hematopoietic cell transplantation (HCT)-specific comorbidity index: a new tool for risk assessment before allogeneic HCT. Blood. 2005;106:2912-9.

15. Shaffer BC, Ahn KW, Hu ZH, Nishihori T, Malone AK, Valcárcel D, et al. Scoring system prognostic of outcome in patients undergoing allogeneic hematopoietic cell transplantation for myelodysplastic syndrome. J Clin Oncol. 2016;34:1864-71.

16. Carré M, Porcher R, Finke J, Ehninger G, Koster L, Beelen D, et al. Transplantationspecific comorbidity index in myelodysplastic patients undergoing an allotransplant: a retrospective study from the Chronic Malignancies Working Party of the European Group for Blood and Marrow Transplantation. Biol Blood Marrow Transplant. 2020;26:451-7.

17. Gauthier J, Damaj G, Langlois $C$, Robin M, Michallet $M$, Chevallier $P$, et al. Contribution of revised International Prognostic Scoring System cytogenetics to predict outcome after allogeneic stem cell transplantation for myelodysplastic syndromes: a study from the French Society of Bone Marrow Transplantation and Cellular Therapy. Transplantation. 2015:99:1672-80.

18. Tamari R, Oran B, Hilden P, Maloy $M$, Kongtim $P$, Papadopoulos EB, et al. Allogeneic stem cell transplantation for advanced myelodysplastic syndrome: Comparison of outcomes between CD34+ selected or unmodified hematopoietic stem cells transplants. Biol Blood Marrow Transplant. 2018;24:1079-87.

19. Barba P, Martino R, Zhou Q, Cho C, Castro-Malaspina H, Devlin S, et al. CD34+ cell selection versus reduced-intensity conditioning and unmodified grafts for allogeneic hematopoietic cell transplantation in patients age $>50$ years with acute myelogenous leukemia and myelodysplastic syndrome. Biol Blood Marrow Transplant. 2018;24:964-72.

20. Malard F, Labopin M, Cho C, Blaise D, Papadopoulos EB, Passweg J, et al. Ex vivo and in vivo $T$ cell-depleted allogeneic stem cell transplantation in patients with acute myeloid leukemia in first complete remission resulted in similar overall survival: on behalf of the ALWP of the EBMT and the MSKCC. J Hematol Oncol. 2018;11:127.
21. Montoro J, Ceberio I, Hilden P, Maloy MA, Barker J, Castro-Malaspina H, et al. Ex vivo $\mathrm{T}$ cell-depleted hematopoietic stem cell transplantation for adult patients with acute myelogenous leukemia in first and second remission: long-term disease-free survival with a significantly reduced risk of graft-versus-host disease. Biol Blood Marrow Transplant. 2020;26:323-32.

22. Urbano-Ispizua A, Brunet S, Solano C, Moraleda JM, Rovira M, Zuazu J, et al. Allogeneic transplantation of CD34+-selected cells from peripheral blood in patients with myeloid malignancies in early phase: a case control comparison with unmodified peripheral blood transplantation. Bone Marrow Transpl. 2001; 28:349-54.

23. Bayraktar UD, de Lima M, Saliba RM, Maloy M, Castro-Malaspina HR, Chen J, et al. Ex vivo $T$ cell-depleted versus unmodified allografts in patients with acute myeloid leukemia in first complete remission. Biol Blood Marrow Transplant. 2013;19:898-903.

24. Hobbs GS, Hamdi A, Hilden PD, Goldberg JD, Poon ML, Ledesma C, et al. Comparison of outcomes at two institutions of patients with ALL receiving ex vivo Tcell-depleted or unmodified allografts. Bone Marrow Transpl. 2015;50:493-8.

25. Cho C, Hsu M, Barba P, Maloy MA, Avecilla ST, Barker JN, et al. Long term prognosis for 1 year relapse free survivors of CD34 cell-selected allogeneic hematopoietic stem cell transplantation: a landmark analysis. Bone Marrow Transpl. 2017;52:1629-36.

26. Barba $P$, Hilden $P$, Devlin SM, Maloy M, Dierov D, Nieves J, et al. Ex vivo CD34+ selected T-cell depleted (TCD) peripheral blood stem cell grafts for allogeneic hematopoietic stem cell transplantation in acute leukemia and myelodysplastic syndrome is associated with low incidence of acute and chronic graft-versus-host disease (GVHD) and high treatment response. Biol Bone Marrow Transplant. 2017;23:452-8.

\section{AUTHOR CONTRIBUTIONS}

AAT and M-AP designed the study. AAT, MM, JDR, MS-E, LY, and NC collected data. $\mathrm{KW}, \mathrm{SD}, \mathrm{AAT}$, and M-AP analyzed and interpreted data. All authors participated in writing the manuscript, and all approved the final manuscript.

\section{FUNDING}

This research was supported in part by National Institutes of Health award number P01 CA23766 and NIH/NCI Cancer Center Support Grant P30CA008748. The content is solely the responsibility of the authors and does not necessarily represent the official views of the National Institutes of Health. AAT is supported by a grant from the Alfonso Martin Escudero Foundation.

\section{COMPETING INTERESTS}

SAG has served as a consultant for Amgen, Actinium, Celgene, Johnson \& Johnson, Jazz Pharmaceutical, Takeda, Novartis, Kite, and Spectrum Pharma and has received research funding from Amgen, Actinium, Celgene, Johnson \& Johnson, Miltenyi, and Takeda. M-AP has received honoraria from Abbvie, Bellicum, Bristol-Myers Squibb, Incyte, Merck, Novartis, Nektar Therapeutics, and Takeda; serves on DSMBs for Servier and Medigene, and the scientific advisory boards of MolMed and NexImmune; and has received research support for clinical trials from Incyte, Kite (Gilead), and Miltenyi Biotec.

\section{ADDITIONAL INFORMATION}

Correspondence and requests for materials should be addressed to M.-A.P.

Reprints and permission information is available at http://www.nature.com/ reprints

Publisher's note Springer Nature remains neutral with regard to jurisdictional claims in published maps and institutional affiliations. 